reducing the incidence of serious injury by taking children off the road.' We agree that the place for the BMX bicycle is off the road, preferably on purpose built tracks. Appropriate training, supervision, and protective clothing should minimise the incidence of injury at low speed. Children riding on the public roads, however, must be encouraged to view BMX bicycles as a vulnerable form of transport.

1 Park KGM, Dickson AP. BMX bicycle injuries in children. Injury 1986;17:34-6.

2 Soysa SM, Grover ML, McDonald PJ. BMX bike injuries: the tatest epidemic. $\mathrm{Br}$ Med $\mathcal{J}$ 1984;289:960-1.

3 Illingworth CM. Injuries to children riding BMX bikes. Br Med f 1984;289:956-7.

(Accepted 23 fanuary 1987)

Department of Traumatic and Orthopaedic Surgery, Cardiff Royal Infirmary, Cardiff CF2 1SZ

S R JOHNSON, MB, FRCSED, registrar in orthopaedics

J A FAIRCLOUGH, MB, FRCS, senior registrar in orthopaedics

Correspondence to: $\mathrm{Mr}$ Johnson.

\section{Controlled trial of $\gamma$ linolenic acid in Dukes's C colorectal cancer}

Colorectal cancer is a common cause of death. This reflects the advanced stage of disease in many patients when the diagnosis is made and the limitations of treatment other than surgery. Cytotoxic drugs do not prevent relapse when given as an adjunct to surgery and do not increase the survival of patients with advanced disease. More effective forms of treatment are needed. Interest has been shown, at least by patients, in a nutritional approach to treatment, and there has been a drift towards centres for alternative treatment of cancer such as those in Bristol and Morecambe Bay, where diet plays a large part in the management programme. Controlled studies of dietary additives in the treatment of cancer have not, however, shown any benefit.

The diet used at the centre in Bristol includes a combination of $\gamma$ linolenic acid and vitamin $E$ (Efamol). $\gamma$ Linolenic acid, an oil extract of the seed from the evening primrose plant (Oenothera lamarckiana), is a polyunsaturated fatty acid from which prostaglandin $E_{1}$ is synthesised in a process requiring

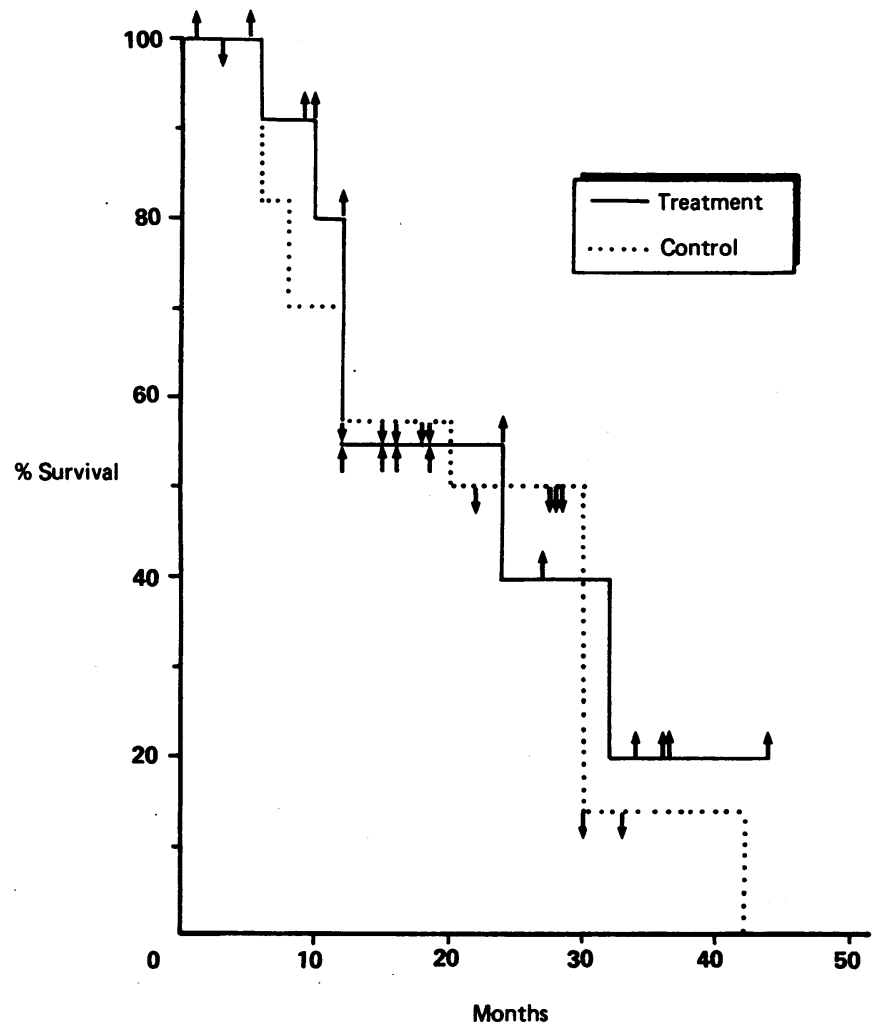

Survival in the two groups of patients. $\uparrow=$ Survivors among treated patients; $\downarrow=$ Survivors among controls. vitamin $C$, pyridoxine, and zinc. ${ }^{1}$ Prostaglandin $E_{1}$ can favourably affect the growth characteristics of animal and human tumour cells maintained in culture. $^{2}$

We have evaluated $\gamma$ linolenic acid in the treatment of colorectal cancer. 3 The dose used was empirical but was considerably more than the dose $\unrhd$ suggested on theoretical grounds. ${ }^{2}$ We selected patients with Dukes's $\mathrm{C} C$ colorectal cancer because the residual tumour mass is small after operation, $\widehat{\widehat{O}}$ the relapse rate is high, and no other effective treatment is available. $\bar{J}$ Moreover, metastases usually appear first in the liver and the highest 70 concentration of $\gamma$ linolenic acid would be in the portal circulation, $\mathbb{D}$ circumstances which should maximise any therapeutic effect.

\section{Patients, methods, and results}

Approval for the study was granted by the local district ethical committee, and informed consent was obtained from each patient. Patients with Dukes's $C$ colorectal cancer were assigned at random within one month of operation to $\frac{\bar{T}}{T}$ receive Efamol capsules containing $\gamma$ linolenic acid $500 \mathrm{mg}$ and natural vitamin $\mathrm{E}$ $10 \mathrm{mg}$ or capsules that were identical in appearance but contained an inert placebo. Six capsules were given daily in divided doses for an indefinite period. \& All patients were also given six compound vitamin tablets daily each containing $\rightarrow$ vitamin $C 125 \mathrm{mg}$, pyridoxine $25 \mathrm{mg}$, and zinc sulphate $5 \mathrm{mg}$. There were 54 . patients in the study. All patients had normal serum carcinoembryonic antigen $\vec{A}$ concentrations, and results of liver function tests were normal. None of the $\vec{\omega}$ patients had any evidence of metastatic disease before admission to the study. One patient stopped treatment after 12 months. Four patients failed to attend after admission to the study. Of the remaining 49 patients, 25 received $\gamma$ linolenic acid and vitamin $E$ and 24 received placebo. Their mean ages were $62 \cdot 1$ years (range 48-81) and 64.8 years (range 45-77) respectively.

The figure shows the survival rate for the two groups. Ten patients in the treatment group died, with a median survival of 12 months (range 6-32); 12 o patients in the control group died, with a median survival of 12 months (range ? 6-42). In all cases death was due to local recurrent or metastatic liver disease or $\vec{N}$ both; there was no difference in the pattern of disease in the two groups. No side $O$ effects of treatment were noted apart from occasional facial flushing, which was attributed to pyridoxine. Twenty seven patients survived, with a median follow up of 20 months (range 1 to 44 months) and 22 months (range 3 to 33 months) in the treatment and control groups, respectively.

\section{Comment}

A nutritional approach to treatment has been shown in limited studies to ? have tumour regulating properties in some types of human cancer but not colorectal cancer. Thus $\gamma$ linolenic acid caused growth regression when added to cultured melanoma, hepatoma, osteogenic sarcoma, and oesophageal cells..$^{3-5}$ Moreover, a striking reduction in liver size has been reported in patients with primary liver cancer taking $\gamma$ linolenic acid, and there is preliminary evidence of a response to $\gamma$ linolenic acid in patients with mesothelioma and astrocytoma (Abstract 161. Second congress of essential fatty acids, prostaglandins and leukotrienes, London 24-27 March, 1985). The mechanism is thought to be as follows: $\gamma$ linolenic acid can bypass the enzyme deficiency, common to cells of many tumour types, of $\delta-6-$ desaturase, which normally converts dietary cis-linolenic acid to $\gamma$ linolenic acid, an essential step in the synthesis of prostaglandin $E_{1}$. This and other prostaglandins have antimetastatic actions and can initiate the process of reverse transformation of tumour cells in culture, thus probably having an important role in modulating cell behaviour. ${ }^{2}$

This is the first reported controlled clinical trial of $\gamma$ linolenic acid in human cancer and it has failed to show benefit in colorectal cancer.

We thank Efamol Ltd, Guildford, Surrey, for supplying the capsules containing $\gamma$ linolenic acid and vitamin $\mathrm{E}$ and the inert capsules.

1 Horrobin DF, Manku MS, Oka M. The nutritional regulation of T lymphocyte function

Med Hypotheses 1979;5:969-85.
Horrobin DF. The reversibility of cancer: the relevance of cyclic AMP, calcium, essential fatty Horrobin DF. The reversibility of cancer: the relevance of cycl
acids, and prostaglandin $E_{1}$. Med Hypotheses 1980;6:469-86.

3 Dippenaar N, Booyens J, Fabbri D, Katzeff IE. The reversibility of cancer: evidence that N malignancy in melanoma cells is gamma linolenic acid deficiency-dependent. $S$ Afr Med $\mathcal{J}$

4 Dippenaar N, Booyens J, Fabbri D, Katzeff IE. The reversibility of cancer: evidence that 0 malignancy in hepatoma cells is gamma linolenic acid deficiency-dependent. $S$ Afr Med $\mathcal{J}$ 1982;62:683-5.

5 Booyens J, Dippenaar N, Fabbri D. The effect of the prostaglandin precursor gamma-linolenic acid : on the rate of proliferation of human osteogenic sarcoma and oesophageal carcinoma cells in culture. S Afr Med F 1984;65:240-2.

(Accepted 13 February 1987)

Royal Lancaster Infirmary, Lancaster LA1 4RR

M B MCILLMURRAY, DM, FRCP, consultant physician

W TURKIE, MB, MRCP, medical registrar

Correspondence to: Dr McIllmurray. , . 